\title{
PREPARATION AND CHARACTERIZATION OF ANDROGRAPHOLIDE NANOPARTICLES FOR VISCERAL LEISHMANIASIS CHEMOTHERAPY: IN VITRO AND IN VIVO EVALUATIONS
}

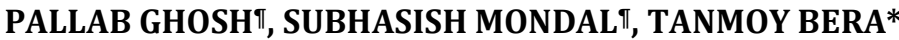 \\ Division of Nanomedicine, Department of Pharmaceutical Technology, Jadavpur University, Kolkata, West Bengal, India \\ Email: proftanmoybera@gmail.com
}

Received: 18 Aug 2016 Revised and Accepted: 05 0ct 2016

\section{ABSTRACT}

Objective: To overcome low physiological solubility, poor bioavailability, the short plasma half-life of andrographolide (AG), a delivery system based on poly (D, L-lactide-co-glycolide) (PLGA) nanoparticles (NPS) were developed to increase the efficiency of AG against visceral leishmaniasis (VL).

Methods: Andrographolide-PLGA nanoparticles (AGnp) were prepared with Pgp efflux inhibitor vitamin E TPGS (D- $\alpha$-tocopheryl polyethylene glycol 1000 succinate) by emulsion solvent evaporation method and characterized. Antileishmanial activity was evaluated using in vitro and in vivo VL infection model.

Results: The particle size of AGnp was found to be171.4 $\pm 11.5 \mathrm{~nm}$ with an encapsulation efficiency of $81 \%$. The AGnp reduced AG cellular toxicity retained it's in vitro antileishmanial activity and lead to a reduction (99.9\%) of parasite burden in the Leishmania donovani infected spleen and liver. AGnp was more active in infected mice liver at low dose than in spleen. Therapeutic indexes (TI) were 6.9-fold greater in AG and 68-fold in AGnp compared to amphotericin B (AmB) when evaluated in L. donovani infected spleen.

Conclusion: Incorporation of AG in PLGA nanoparticles, provided controlled and improved in vivo performance against VL

Keywords: Andrographolide, PLGA nanoparticles, In vitro activity, In vivo antileishmanial activity, Macrophage, Therapeutic index

(C) 2016 The Authors. Published by Innovare Academic Sciences Pvt Ltd. This is an open access article under the CC BY license (http://creativecommons.org/licenses/by/4. 0/)

DOI: http://dx.doi.org/10.22159/ijpps.2016v8i12.14773

\section{INTRODUCTION}

Leishmaniasis is a neglected tropical disease, indigenous to tropical and subtropical areas and affects about 12 million people around the world. However, it's also becoming more common in developed countries, because of the increasing immunosuppressed population [1]. The current therapy against this parasite infection is not adequate and has the following limitations: toxicity, high cost, noneffectiveness, resistance or hospitalization requirement. The first line chemotherapy treatments are based on injectable pentavalent antimonials (Pentostam $®$ and Glucantime ${ }^{\circledR}$ ), which are highly toxic and drug resistance has already proved to be an issue [2].

AmB deoxycholate (Fungizone $\AA$ ) normally considered as secondline drug, which has become the first line therapy in Bihar, India. Following the loss of effectiveness of antimonial drugs, it requires careful and slow intravenous administration due to its high toxicity [3]. Liposomal AmB formulations (Ambisome®) have been developed in order to improve toxicological and pharmacokinetic properties. Virtually, these lipidic formulations reduced toxicity exhibited a better half-life and a higher efficacy in the leishmaniasis treatment. Unfortunately, their high cost and relapse in immunocompetent patients are the major limitations for their largescale use especially in under developing countries [4].

Oral antineoplastic agent, miltefosine (MIL) was introduced as the first effective oral treatment for VL, and as an alternative treatment for HIV patients [5]. Teratogenic potential and resistance development are the major limitations of MIL in women of childbearing age $[5,6]$. A parenteral formulation of aminoglycoside antibiotic paromomycin (PMM), showed 94\% efficacy in clinical trials and introduced for VL in India [7]. A strategy of coadministration of available antileishmanial drugs was carried out by the drugs for Neglected Diseases initiative to provide safety and efficacy for the treatment of VL [8].

It is roughly estimated that of the discovered 17,000 species of plants, nearly 3,000 species are used in the medicinal field. The use of plant extracts and phytochemicals, both with known antimicrobial properties, can be of great significance in therapeutic treatments [9]. Numerous plant derived products from different structural classes have been investigated as antileishmanial candidates including various alkaloids, terpenoids, flavonoids and quinonoid. AG, a labdane diterpene was isolated from the leaves of Indian medicinal plant Andrographis paniculata, exert significant antiproliferative effects on the life cycle of $L$. donovani, the causative agent of VL [10, 11]. However, this compound has drawbacks, such as low aqueous solubility, poor bioavailability, and short plasma half-life, making their potential development as chemotherapeutic agent prohibitive. By using Nano Drug Delivery System (NDDS), one can reduce their cytotoxicity side effects, site-directed drug delivery, and aqueous solubility properties, hence contributing to their bioavailability.

Leishmania parasites are dimorphic, alternating between promastigote and amastigote form during their life-cycle. In macrophage cells of mammals, Leishmania cells reside and multiply [12]. Fibroblasts play an important role during the chronic phase of parasitic infection due to their inability to sustain parasite killing and may provide a safe target for the parasites in clinically latent diseases [13]. Indeed, phagolysosomal localization of the parasites, prevent drugs from readily diffusing into the target and hence represents a major problem in the development of effective antileishmanial agent. Therefore, the use of NDDS, capable of delivering antileishmanial compounds to infected cells should improve the therapeutic efficacy (therapeutic index,) of these drugs and at the same time, reduce the toxicity by altering the pharmacokinetic and bio distribution profile of the drug.

Many NDDS have already been used for antileishmanial compounds [14]. In general, these systems tend to increase drug efficacy while decreasing toxicity, resulting in an improved therapeutic index (TI) [15]. The biggest improvement, the cost-efficacy benefit, is believed to come from nano drug carriers. Recently, polylactide NPs containing basic acid were more effective than microemulsions in an in vivo hamster model of $\mathrm{VL}$ [16]. Other example includes AmBloaded in poly ( $\varepsilon$-caprolactone) NPs [17] or in mannose-anchored, PLGA NPs for the efficient delivery of AmB to macrophages [18]. 
The aim of this study was to develop and characterize a controlled drug delivery system for the treatment of VL, using an in vitro active AG [10, 11]. Therefore, hydrophobic $A G$ was encapsulated in the biodegradable polymer PLGA, in order to increase its solubility, reduce cytotoxicity and enhance TI. The physicochemical characterization, the biological evaluation and its delivery system of this new antileishmanial drug were investigated. The AG load PLGA NPs were able to reduce cellular drug toxicity, retaining it's in vitro antileishmanial activity and improving efficacy by 8 to 10 fold when compared with free AG on L. donovani infected BALB/c mice, in the spleen.

\section{MATERIALS AND METHODS}

\section{Animal and ethics statement}

Six-week-old BALB/c mice of either sex, weighing 20-25 g and of approximately the same age were used for the study and they were obtained from a biological supply vendor (Rita Ghosh and Co., Kolkata, India). The experimental protocols were approved by the Jadavpur University Animal Ethics Committee (Id \# JU/Pharm./384/11), and procedures followed were in accordance with the Committee for the Purpose of Control and Supervision of Experiments on Animals (CPCSEA) of Government of India and followed the standards as described in the Guide for the Care and Use of Laboratory Animals. Animals were housed five per cage for acclimatization one week before experiments at the animal house facility of Jadavpur University. Mice were fed with a standard diet and water ad libitum. Mice were exposed to a normal day and night cycle.

\section{Experimental materials: medicines and chemical reagents}

PLGA (lactide: glycolide $=50: 50$, molecular weight: 40,000-75000 Da), TPGS, $\mathrm{C}_{33} \mathrm{O}_{5} \mathrm{H}_{54}\left(\mathrm{CH}_{2} \mathrm{CH}_{2} \mathrm{O}\right)_{23}, \mathrm{AG}, \mathrm{PMM}, \mathrm{AmB}$, MIL, fluorescein 5(6)-isothiocyanate (FITC), medium 199, RPMI 1640, dialysis tubing D9652 (MW cut of 12400) and fetal calf serum (FCS) were purchased from Sigma-Aldrich (USA). Sodium stibogluconate (SSG) was a generous gift from Albert David Ltd. (Kolkata, India). All other chemicals and reagents were reagent grade or higher.

\section{Equipment and instruments}

Standard glassware of Borosil ${ }^{\circledR}$ were used for experimental purpose. A 700 MW sonicator, model vibra cell vex 750 (Sonics, USA); homogenizer, model TH 02 (Omni International, USA); a precision balance $0.00001 \mathrm{~g}$, Mettler Toledo AL54 (Mettler, USA); an ultracentrifuge, Himac CS 120 GHXL (hitachi Koki, Japan) were used in the preparative processes. Chemical analysis were carried out by dual pump HPLC model (UHPLC+, Dionex UltiMate 3000, Thermo Scientific); Zetasizer nano ZS (Malverm, UK); atomic force microscope nanoscope 3A (Veeco, England); FEI Tecnai TM Transmission Electron Microscopy (Netherland) and fluorescence microscope (B X 51, Olympus, USA) were used for particle characterization and imaging experiments. GraphPad Prism 5.01 and Sigmaplot 6.0, (Zendal Scientific, USA) were used for the data analysis purposes.

\section{Preparation of PLGA NPs containing AG}

The encapsulation of AG in PLGA NPs was obtained by emulsion solvent evaporation method as described previously by Roy et al. [10]. Briefly, $100 \mathrm{mg}$ PLGA and $5 \mathrm{mg}$ of AG were dissolved in $6 \mathrm{ml}$ chloroform. This phase was emulsified by sonication for $5 \mathrm{~min}$ in $0.04 \%(\mathrm{w} / \mathrm{v})$ of aqueous TPGS solution at $20 \mathrm{KHz}$. Resultant o/w emulsion was homogenized for $30 \mathrm{~min}$ at 20000 r. p. m under external cooling in ice water. The solvent evaporation was continued for $12 \mathrm{~h}$ over a magnetic stirrer at room temperature. An initial centrifugation at $5000 \mathrm{xg}$ for $15 \mathrm{~min}$ at $4{ }^{\circ} \mathrm{C}$ was performed to remove larger particle aggregates. The resulting supernatant was recovered and centrifuged at 30000 r. p. m. for $25 \mathrm{~min}$ at $4{ }^{\circ} \mathrm{C}$. AGnps were washed with water, recentrifuged and preserved after decontamination by $30 \mathrm{~min} \mathrm{UV}$ exposure, at $-20^{\circ} \mathrm{C}$ up to $6 \mathrm{mo}$. Stability of NPs in terms of morphology and drug content was not affected either by storage at $-20^{\circ} \mathrm{C}$ or the decontamination process (data not shown).

\section{Preparation of fluorescein-loaded PLGA NPs (FITCnp)}

FITC was used as a model drug and was encapsulated into PLGA NPs by emulsion solvent evaporation method described above. FITC was dissolved in dimethylformamide (DMF) at $100 \mathrm{mg} / \mathrm{ml}$ and $0.1 \mathrm{ml}$ of solution was added into $10 \mathrm{mg}$ of polymer in $2 \mathrm{ml}$ of chloroform, and rest of the process was performed as described above.

\section{Nanoparticle characterization: size, polydispersity and $\zeta$ potential}

Frozen NPs were diluted in distilled water at $1 \mathrm{mg} / \mathrm{ml}$. NPs were characterized in terms of size, polydispersity, and surface potential by dynamic light scattering with a $4 \mathrm{~mW}$ He-Ne laser beam of 633 $\mathrm{nm}$ wavelength (Zetasizer, Melvern Instruments). A minimum of three different sets was examined at $25^{\circ} \mathrm{C}$, with a detection angle of $173^{\circ}$ using NIBS technology.

\section{Morphology}

The morphology of NPs was observed by transmission electron microscopy (TEM: JEM-1400, Jeol Tokyo, Japan) and atomic force microscopy (AFM: Veeco Nanoscope IIIa, England). AGnp suspension $(100 \mu \mathrm{l})$ in water were deposited into fused mica substrates by drop cast method. The particles were visualized in AFM in tapping mode using RTESP tip with $267328 \mathrm{KHz}$ resonance frequency at a scan speed of $1.2 \mathrm{~Hz}$. The morphology of NPs examined by the conventional negative staining method, operated with an accelerated voltage of $80 \mathrm{kV}$. One drop of NPs was placed over the carbon-coated copper grid, and then that grid was washed with distilled water. Then staining was performed with uranil acetate solution. The sample was air-dried before observation.

\section{AG encapsulation efficiency and drug loading}

AG nanoformulations were evaluated in terms of encapsulation efficacy (EE), according to equation (1).

EE (\%)

$$
=\frac{\text { Mass of AG originally taken }- \text { Mass of AG in supernatant }}{\text { Mass of AG originally taken }} \times 100
$$

Estimation of AG in all cases was carried out by a reverse phase HPLC system. The mobile phase was acetonitrile $(0.1 \% \mathrm{v} / \mathrm{v})$ and phosphoric acid in water $(40: 60 \mathrm{v} / \mathrm{v})$ at a flow rate of $1 \mathrm{ml} /$ minutes. The analysis was carried out by using $250 \mathrm{X} 4.6 \mathrm{~mm} \mathrm{C}_{18}$ column. A peak area (y) vs. concentration $(\mathrm{x})$ graph for AG was first prepared, $\mathrm{y}=30145, \mathrm{x}=$ $68911, \mathrm{R}^{2}=0.9930$, retention time was $4.5 \mathrm{~min}$. This was used to detect AG concentration throughout. Mass of AG in solution before and after nanoparticulation in the supernatant was determined by HPLC experiments for calculation of entrapment efficiencies [11].

\section{In vitro drug release assay}

The drug-loaded NPs were dispersed in phosphate buffered saline (PBS) (0.1 M, pH 7.4 or pH 5.5) and were then placed in an orbital shaker, shaking at 300 r. p. m. at $37^{\circ} \mathrm{C}$. At designated time intervals, the suspension was centrifuged at $30000 \mathrm{r}$. p. m. for $20 \mathrm{~min}$. The pellet was resuspended in the corresponding fresh buffer to continue the drug release, and the supernatant was used for drug quantification. The quantity of AG was determined by the same HPLC procedure as mentioned above. The error bars were obtained from the triplicate samples.

\section{Cell culture}

The mice peritoneal monocyte cells were grown at $37{ }^{\circ} \mathrm{C}$ with $5 \%$ $\mathrm{CO}_{2}$ in RPMI 1640 medium, complemented with $10 \%$ heatinactivated fetal bovine serum, $2 \mathrm{mmol}$ L-glutamine, $100 \mathrm{U} / \mathrm{ml}$ penicillin and $100 \mu \mathrm{g} / \mathrm{ml}$ streptomycin. For macrophage differentiation, mice monocyte cells were incubated in the presence of $20 \mathrm{mg} / \mathrm{ml}$ phorbal 12-myristate 13-acetate (PMA, Sigms-Aldrich) for $18 \mathrm{~h}$ at $37^{\circ} \mathrm{C}$ and left another $24 \mathrm{~h}$ with fresh medium containing no PMA to induce maturation.

\section{Cellular uptake of FITC-loaded NPs}

In order to study nanoparticle uptake in macrophage cells, the desired cell concentration ( $3 \times 10^{5}$ cells/well) was seeded with FITCnp $(0.5 \mathrm{mg} / \mathrm{ml})$. After incubation of specific times in $\mathrm{CO}_{2}$ incubator at $37^{\circ} \mathrm{C}$, cells were washed twice with PBS to eliminate unbound NPs, centrifuged (1000 r. p. m., 5 min) after trypsinization, then suspended in $0.5 \mathrm{ml}$ of PBS and observed in FITC channel under fluorescence microscope. 


\section{Parasite and culture conditions}

L. donovani AG83 (MHOM/IN/83/AG83) was VL isolate obtained as a gift from Indian Institute of Chemical Biology, Council of Scientific and Industrial Research, Kolkata, India. L. donovani AG83 promastigotes were grown at $25^{\circ} \mathrm{C}$ in medium 199 supplemented with $10 \%$ heat-inactivated fetal bovine serum, $2 \mathrm{mmol}$ L-glutamine, $20 \mathrm{mmol} \mathrm{Hepes,} 100 \mathrm{U} / \mathrm{ml}$ penicillin and $100 \mu \mathrm{g} / \mathrm{ml}$ streptomycin. $L$. donovani axenic amastigotes were derived from promastigotes by culturing them in MMA/20, pH 5.5 (medium for axenic amastigotes) culture medium as described previously [10]. Amastigotes were maintained in culture, at $37{ }^{\circ} \mathrm{C}$ in an atmosphere of $5 \% \mathrm{CO}_{2}$ by subpassage $\left(10^{5}\right.$ parasites $\left./ \mathrm{ml}\right)$, every $5 \mathrm{~d}$.

\section{Evaluation of drug susceptibility on intracellular $L$. donovani amastigotes}

The growth of the AG83 wild-type amastigotes on the mouse macrophages was evaluated as described [11]. Briefly, adherent cells grown in 24-well plates at $37{ }^{\circ} \mathrm{C}$ with $5 \% \mathrm{CO}_{2}$, were incubated with stationary-phase promastigotes for $4 \mathrm{~h}$ at $37^{\circ} \mathrm{C}$ at a $1: 5$ cell: parasite ratio. After the parasite internalization period, cells were washed at least twice with fresh media to remove non-phagocytosed parasites and kept with variable concentrations of free AmB, SSG, PMM, MIL, AG or AGnp for $72 \mathrm{~h}$. The amastigote number in macrophages was assessed after staining with Giemsa, 100 cells on the glass disks were counted by inverted microscope. Three independent experiments in triplicate for each concentration were performed for the efficacy of drugs and NPs. Results were presented as the ratio between the infection proportions of treated and untreated macrophage cells. Drug concentration necessary to decrease the number of intracellular amastigotes to $50 \%$, inhibitory concentration $50 \%\left(\mathrm{IC}_{50}\right)$, was determined by linear regression analysis.

\section{Cytotoxicity assay and selectivity index}

Macrophages cells were cultured in RPMI-1640 supplemented with $10 \%$ FCS, 20 mmol L-glutamate, $16 \mathrm{mmol} \mathrm{NaHCO3}$, penicillin (50 $\mathrm{U} / \mathrm{ml})$ and streptomycin $(50 \mu \mathrm{g} / \mathrm{ml})$. The assay was performed in 24-well tissue culture plates in the presence of standard counts of macrophages. The wells were seeded with test solutions, and the viable macrophages were counted microscopically.

A key part of drug discovery and development is the characterization and optimization of the safety and efficacy of drug candidates and to identify those that have an appropriately balanced safety-efficacy profile for a given indication. The selectivity index (SI) which is typically considered as the highest exposure to the drug that results in no toxicity to the exposure that produces the desired efficacy, is an important parameter in efforts to achieve this balance. In the present study, the degree of selectivity of the drug or its formulation is expressed as $\mathrm{SI}=$ cytotoxicity $\left(\mathrm{CC}_{50}\right)$ of a drug or its formulation in a macrophage cell line/IC ${ }_{50}$ of the same drug or its formulation, where $\mathrm{CC}_{50}$ is the concentration required to kill $50 \%$ of the host cell population and $\mathrm{IC}_{50}$ is the concentration required to kill $50 \%$ of the parasites inside the host cell. When the SI value is $\geq 10$, that drug or formulation will present promising activity, that is higher than its $\mathrm{CC}_{50}[19]$.

\section{In vivo studies}

For infection of mice, stationary phase promastigotes of AG83 wildtype were collected, washed and suspended with sterile PBS. A volume of $200 \mu \mathrm{l}$ of sterile PBS containing $10^{7}$ parasites was injected through tail-vain, which will lead to a systemic distribution of parasite throughout the body. The mice from all infected groups were treated after $45 \mathrm{~d}$ of infection by intraperitoneal injection of $200 \mu \mathrm{l}$ membrane sterilized drug solutions or nanoformulation. Dosing schedule was either one dose/day for 5 consecutive days or one dose/week. Each dose was given to 5 mice. Treatment groups were as follows:

Group I: Infected and drugs or NPs untreated negative control;

Group II: Infected and treated with drugs or NPs solution as positive control;

Group III: Uninfected and drugs or NPs untreated control.

Seven days after the last treatment administration, the animals were euthanized and their spleens and livers were aseptically removed for parasite quantification by limiting dilution, as described [20].

\section{Statistical analysis}

Experimental results were expressed as mean \pm standard deviation. Student's t-test was used to calculate the statistical difference of mean values. Differences were considered significant at a level when $\mathrm{p}<0.01$. For toxicity studies, an unpaired t-test was used to compare groups. A p value $<0.05$ was considered to be statistically significant.

\section{RESULTS}

The physicochemical characteristics of the formulated nanoparticles such as particle size, polydispersity, $\zeta$-potential and drug encapsulation efficiency are summarized in table 1 . Among the various physicochemical properties of NPs, the drug loading, particle size and surface property may be the most important parameters that determine theirs in vitro and in vivo performance [15]. The NPs were prepared from the PLGA by emulsification solvent diffusion method. The optimized amount of $0.04 \%(\mathrm{w} / \mathrm{v}$ ) of TPGS used as an emulsifier exhibited negative surface charge $(-35.8 \mathrm{mV})$ indicating near stable dispersion of NPs with a narrow size distribution $(171.4 \pm 11.5 \mathrm{~nm})$ identical to unloaded PLGA NPs (168.5 $\pm 10.3 \mathrm{~nm}$ ) (table 1).

The polydispersity index characterizes the nanoparticulate system in terms of size homogeneity by a value of the relative variance of sizes in a NP population [21]. The characterization of the unloaded and AG loaded NPs by TEM and AFM (fig. 1 and 2, respectively) showed a monodispersed size distribution and good redispersibility suggesting that their surface is stabilized by the deposition of TPGS, which prevent aggregation. Before release study, AGnp showed no change or perforation at the surface (fig. 2A). Whereas AGnp showed cleavage perforation at the surface (fig. 2B) after $600 \mathrm{~h}$ release study.

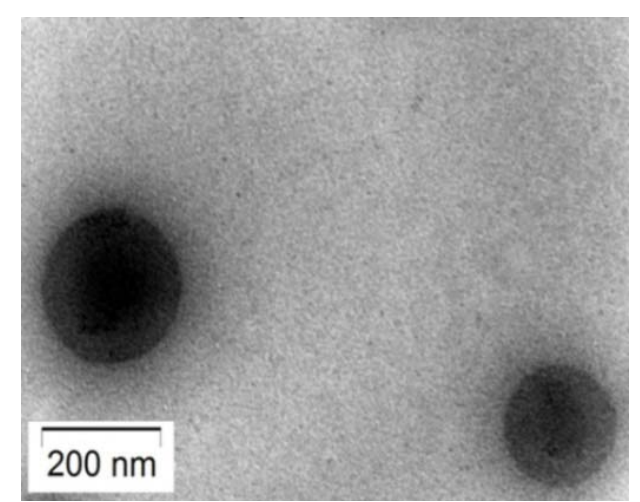

Fig. 1: TEM micrograph of AGnps with TPGS obtained from the emulsion solvent evaporation method. One drop of NPs was placed over the carbon-coated copper grid. The sample loaded copper grid was washed with distilled water. Then staining was performed with uranil acetate solution and the sample was air

dried before observation. Magnifications: 30000X and acceleration voltage $80 \mathrm{kV}$

Table 1: Size, polydispersity index and $\zeta$-potential characterization of nano-formulations ${ }^{\mathrm{a}}$

\begin{tabular}{|c|c|c|c|c|}
\hline $\begin{array}{l}\text { Nanoformulat } \\
\text { ions }\end{array}$ & $\begin{array}{l}\text { Size } \\
(\mathrm{nm})\end{array}$ & $\begin{array}{l}\text { Polydispersi } \\
\text { ty index }\end{array}$ & $\begin{array}{l}\zeta- \\
\text { potent } \\
\text { ial } \\
(\mathrm{mV})\end{array}$ & $\begin{array}{l}\text { Encapsula } \\
\text { tion } \\
\text { efficiency } \\
(\%)\end{array}$ \\
\hline Empty PLGA & $168.5 \pm$ & $0.24 \pm 0.03$ & - & NA \\
\hline NPs & 10.3 & & $\begin{array}{l}38.3 \pm 1 \\
7\end{array}$ & \\
\hline AGnps & $\begin{array}{l}171.4 \pm \\
11.5^{*}\end{array}$ & $0.23 \pm 0.02$ & $\begin{array}{l}- \\
35.8 \pm 1 \\
5\end{array}$ & $81.1 \pm 3.4$ \\
\hline
\end{tabular}

aResults are expressed as mean \pm SEM, $(n=4) .{ }^{*} \mathrm{p}<0.001$, statistically significant difference when compared with empty NPs. NA: Not Applicable. 

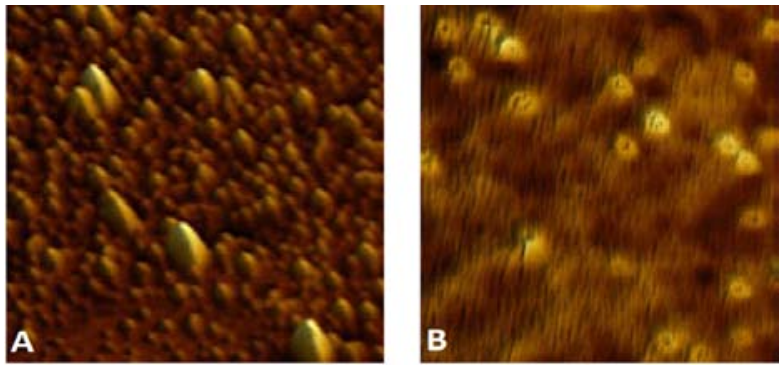

Fig. 2: Atomic force microscopy of AGnp before and after dissolution. A) AGnp before release, B) AGnp $600 \mathrm{~h}$ after AG release. AGnp showed no change or perforation at the surface (fig. 2A). Whereas after $600 \mathrm{~h}$ AGnp showed cleavage perforation at the surface (fig. 2B) indicating release of AG from AGnp

Furthermore, storage stability studies revealed that the physical structure of the NPs is stable up to $3 \mathrm{mo}$ at $4{ }^{\circ} \mathrm{C}$ [11]. The release profile of AGnps under physiological (7.4) pH and acidic (5.5) $\mathrm{pH}$ (similar to the phagolysosome) are shown in fig. 3.

It was found that a significant initial burst release profile was obtained for both conditions during the first half day. Especially, in the case of $\mathrm{pH} 5.5$, AGnps released approximately $50 \%$ of its content over the first day of the study, while under the physiological $\mathrm{pH}$ the same quantity was released after $1 \mathrm{w}$. After the initial burse release, there was a phase of constant AG release. The AG release from PLGA NPs reached a plateau after $150 \mathrm{~h}$. We have investigated the in vitro antileishmanial activity of standard antileishmanial drug; AmB, SSG, PMM and MIL along with free AG and AGnp against intracellular wild-type $L$. donovani amastigote cells and compared their $\mathrm{CC}_{50}$ in macrophage cells (table 2).

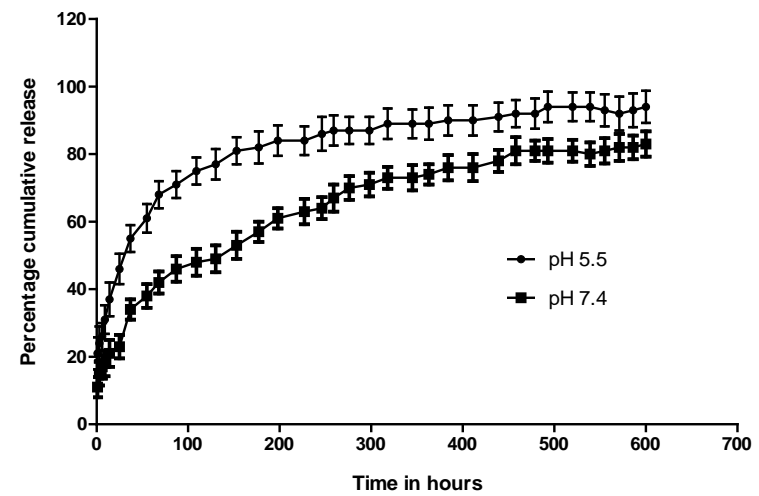

Fig. 3: Release profile of AGnp under physiological pH (7.4) and phagolysosomal pH (5.5). In vitro release profile of AGnp at pH 7.4 $\left(-\square^{-}\right)$and $\left.\mathrm{pH} 5.5(-)^{-}\right)$. Results expressed as mean $\pm \mathrm{SD}(\mathrm{n}=3)$

Table 2: Antileishmanial activity profile of standard antileishmanial drugs, AG and AGnp against intracellular L. donovani wild-type amastigote cell line

\begin{tabular}{llll}
\hline $\mathbf{I C}_{\mathbf{5 0}} \boldsymbol{\mu \mathbf { M } ^ { \mathbf { a } }}$ & & & \\
\hline Drug & Cellular AG83 evaluation model & $\mathbf{C C}_{\mathbf{5 0}} \boldsymbol{\mu} \mathbf{M}$ (Macrophage cells) \\
\cline { 2 - 3 } & Wild-type & 93 & $13 \pm 2.1^{* *}$ \\
\hline AmB & $0.14 \pm 0.05^{*}$ & 17 & $25 \pm 3.9$ \\
SSG $(\mathrm{SbV})^{\mathrm{b}}$ & $1.5 \pm 0.20$ & 34 & $235 \pm 32^{*}$ \\
PMM & $7 \pm 2^{*}$ & 97 & $33 \pm 6^{*}$ \\
MIL & $0.34 \pm 0.04^{*}$ & 7.1 & $950 \pm 160^{*}$ \\
AG & $134 \pm 20^{*}$ & 73 & $1900 \pm 300^{*}$ \\
AGnp & $26 \pm 2^{*}$ & & \\
\hline
\end{tabular}

Results are expressed as mean $\pm \mathrm{SD},(\mathrm{n}=4)^{\mathrm{a}}$ Assays are described in Materials and Methods, bValues for antimonial agents are in $\mu \mathrm{g} \mathrm{SbV} / \mathrm{ml},{ }^{\mathrm{c} S I}$, Selectivity Index was $\mathrm{CC}_{50} / \mathrm{IC}_{50}$. ${ }^{*} \mathrm{p}<0.001$, significant difference compared with $\mathrm{SSG},{ }^{* *} \mathrm{p}<0.5$, no significant difference compared with SSG.

Using a homogenization solvent evaporation technique, FITC-loaded PLGA NPs were obtained with $173 \pm 10.7 \mathrm{~nm}$ and- $36 \pm 1.8 \mathrm{mV}$, characteristics identical to AGnp, and those were used to evaluate cellular uptake of FITCnp by macrophage cells. Fluorescence microscopy was applied to confirm the internalization of the FITCloaded PLGA NPs by macrophages (fig. 4).
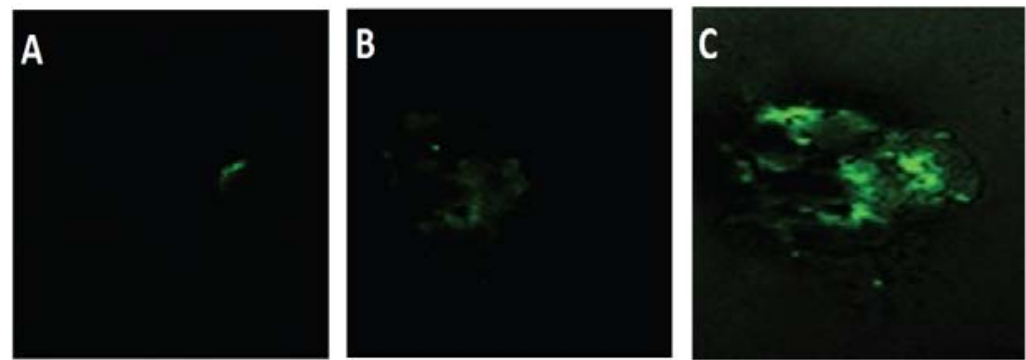

Fig. 4: AGnp uptake in macrophage cells. Fluorescence image. FITCnp were used to study the cellular uptake of FITCnps in macrophage cells as the amastigote cells reside within the phagolysosomes of macrophages. $4 \times 10^{5}$ macrophage cells/well in three batches were studied with FITCnps $(0.5 \mathrm{mg} / \mathrm{ml})$ and incubated in $\mathrm{CO}_{2}$ incubator at $37^{\circ} \mathrm{C}$. For (A), $15 \mathrm{~min}$ (B), $30 \mathrm{~min}$; and (C), 60 min. Samples were

withdrawn after specific time intervals, washed and observed in FITC channel under a fluorescence microscope. FITCnps showed considerable cellular internalization within $60 \mathrm{~min}$ of incubation and insignificant internalization within 15 min. Cellular internalization of FITCnps only took place in viable cells. Fig. represented time-dependent uptake of FITCnps

Macrophage cells under investigation exhibited increasing FITC fluorescence intensity from $15 \mathrm{~min}$ to $60 \mathrm{~min}$. In the present study, the effect of AG and AGnp were evaluated using the acute murine model of VL by treating the mice at $6 \mathrm{w}$ post infection with AG, AGnp and AmB or SSG as the positive control (table 3 and 4). 
Table 3: Comparison of antileishmanial effects of free AG and AGnp on the parasite burden of wild-type L. donovani infected mice

\begin{tabular}{|c|c|c|c|c|}
\hline \multicolumn{5}{|c|}{ \% Suppression of amastigote burden } \\
\hline \multirow[t]{2}{*}{ Drug } & \multirow[t]{2}{*}{ Dose, ip mg/kg/week } & \multirow[t]{2}{*}{ Dosing (number of week) } & Spleen & Liver \\
\hline & & & Wild-type & Wild-type \\
\hline SSG (SbV) & 40 & 4 & $82 \pm 13$ & $93 \pm 2$ \\
\hline$A G$ & 2 & 2 & $23 \pm 3^{*}$ & $24 \pm 4^{*}$ \\
\hline $\mathrm{AG}$ & 2 & 4 & $36 \pm 5^{*}$ & $38 \pm 7 *$ \\
\hline AGnp & 2 & 2 & $84 \pm 12^{* * *}$ & $91 \pm 4^{* * *}$ \\
\hline AGnp & 2 & 4 & $99.9 \pm 0.1^{* *}$ & $99.9 \pm 0.1^{* * *}$ \\
\hline
\end{tabular}

Results are expressed as mean \pm SEM, $(n=5)$. ip: intraperitoneal, ${ }^{*} \mathrm{p}<0.001$, significant difference compared with $\mathrm{SSG}$, ${ }^{* *} \mathrm{p}<0.5$, no $\mathrm{s}$ significant difference compared with SSG, ${ }^{* * *}$ p $>0.5$, no significant difference compared with SSG.

Table 4: Antileishmanial activity profile of standard antileishmanial drugs, AG and AGnp on the parasite burden of $L$. donovani wild-type amastigote cell line in mice

\begin{tabular}{|c|c|c|c|c|}
\hline \multirow{3}{*}{$\begin{array}{l}\mathrm{ED}_{50} \mathrm{mg} / \mathrm{kg}^{\mathrm{a}} \\
\text { Antileishmanial drug and nanoformulation }\end{array}$} & \multirow{3}{*}{ Route of administration (consequtive dose) } & \multirow{2}{*}{\multicolumn{2}{|c|}{ AG83 evaluation model }} & \multirow{3}{*}{$\mathrm{LD}_{50}(\mathrm{mg} / \mathrm{kg})^{\mathrm{b}}$} \\
\hline & & & & \\
\hline & & Wild-type & TI $^{\mathrm{c}}$ & \\
\hline $\mathrm{AmB}$ & $5 \times$ i.p $p$ & $0.3 \pm 0.06^{*}$ & 90 & $27 \pm 7 *$ \\
\hline SSG $(\mathrm{SbV})^{\mathrm{d}}$ & $5 \times$ i. p & $9.5 \pm 2.21$ & 8.9 & $85 \pm 23$ \\
\hline $\mathrm{AG}$ & $5 \times$ i.p & $17 \pm 4.32^{* *}$ & 618 & $10500 \pm 2420^{*}$ \\
\hline AGnp & $5 \times$ i. p & $3.6 \pm 0.77 *$ & 6111 & $22000 \pm 5860^{*}$ \\
\hline
\end{tabular}

Results are expressed as mean $\pm \mathrm{SEM},(\mathrm{n}=5)$, aDetermination of $50 \%$ suppression of parasite burden in spleen of mice by drugs $\left(\mathrm{ED}_{50}\right)$ are described in Materials and Methods and defined as the dose that produces a quantal effect in $50 \%$ of the population that takes it., bDetermination of $50 \%$ death of mice by drugs $\left(\mathrm{LD}_{50}\right)$ are described in Materials and Methods., cTherapeutic Index (TI) is a quantitative measurement of the relative safety of drugs, which is the ratio of $\mathrm{LD}_{50} / \mathrm{ED}_{50}$., dValues for sodium stibogluconate are in $\mathrm{mg} \mathrm{SbV/kg} \mathrm{as} \mathrm{given} \mathrm{in} \mathrm{Materials} \mathrm{and} \mathrm{Methods.,} \mathrm{*p}<0.001$, significant difference compared to stibogluconate., ${ }^{* *} \mathrm{p}<0.5$, no significant difference compared to stibogluconate.

Weekly intraperitoneal administration of all the treatments showed a significant $(\mathrm{p}<0.001)$ lower parasite burden in the visceral organs, spleen and liver when compared with the mice in control groups that received either PBS/DMF or empty PLGA NPs (table 4).

\section{DISCUSSION}

The multidisciplinary field of nanotechnology is bringing the science of the almost incomprehensibly small device closer and closer to reality. Nanotechnology offers the ability to build large numbers of products that are incredibly powerful by today's standards [22].

VL can be caused either by L. donovani (in Asia and Africa) or $L$. infectum (in southern Europe). In this study, L. donovani was selected for the development of the new antileishmanial nanoformulation, since it has been the objective of our research. For the therapeutic application of an antileishmanial agent, high selectivity compared to its host cell is the primary requirement. Selectivity is expressed in terms of selectivity index (SI). To be a safe drug, SI should be greater than 10 [19]. It appears from table 2 that AmB and MIL followed by AGnp were most selective for wild AG83 strains when evaluated in vitro in intracellular amastigotes. The AGnp have demonstrated significant advantages compared to free $\mathrm{AG}$ in reducing cellular toxicity $\left(\mathrm{CC}_{50}\right)$ and increasing SI. These results are in accordance with other in vitro and in vivo studies, in which the encapsulation of a compound into a nanocarrier was proven effective in reducing the toxicity of the drug [13, 15]. The polydispersity index values being less than 0.3 (table 1), indicating that the NPs were of relatively uniform size. The encapsulation of AG into the NPs, resulted in a statistically significant $(\mathrm{p}<0.01)$ decrease in the negative surface charge (table 1) indicating in vitro stable dispersion of NPs. In general, it is thought that NP suspension with large positive or negative $\zeta$-potential values $₫ 30 \mathrm{mV}$ ) are less likely to suffer from aggregation phenomena due to electrical repulsion. Upon the in vivo administration, the physiological condition will mask the almost neutral surface charges of the nanoformulations, contributing to the equilibrium of the system. Drug loading of the nanoformulations was optimized to control the size of NPs. Due to the fact that the amount of encapsulated drug is crucial in a drug delivery system, high drug loading rate is desirable to reach therapeutic necessities. Optimized AGnp was prepared with a drug loading of $8 \%(\mathrm{w} / \mathrm{w})$ and an encapsulation efficiency of 81.1 \pm 3.4 . The high encapsulation efficiency obtained is a consequence of hydrophobic nature of the AG. The treatment with the AGnp was significantly more effective than free AG in reducing parasite burden in spleen and liver, at an equivalent drug dose of 2 $\mathrm{mg} / \mathrm{kg} /$ week either for $2 \mathrm{w}$ or $4 \mathrm{w}$ for wild-type L. donovani. Most strikingly, AGnp at $2 \mathrm{mg} / \mathrm{kg} /$ week dose for $4 \mathrm{w}$ showed $99.9 \%$ lowering of parasite burden in both spleen and liver infected with wild-type $L$. donovani. Therapeutic applicability of any chemotherapeutic agent depends on its safety which is quantitatively expressed as a therapeutic index (TI). TI is the ratio of $\mathrm{LD}_{50} / \mathrm{ED}_{50} . \mathrm{LD}_{50}$ is defined as $50 \%$ death of mice by drug and $\mathrm{ED}_{50}$ as the dose that produces $50 \%$ suppression of parasite burden. TI of free AG and AGnp was determined considering AmB and SSG as a control group in 5 consecutive i. p. dosing with variable drug or nanoformulation concentrations (table 4). For this purpose ED 50 of $\mathrm{AG}$ and AGnp was determined to compare with an infected untreated animal in spleen burden of wild-type L. donovani infected mice and $\mathrm{AmB}$ and stibogluconate as a positive control. Comparing $\mathrm{AmB}$, stibogluconate, and AG, AGnp was clearly more safe and active for wild-type $L$. donovani infected animals. It appears from table 4 that TI of AGnp was 68-fold in wild L. donovani infected mice when compared with AmB, a first line drug of choice for Kala-azar (VL). TI of stibogluconate was less than 10 for wild strains of L. donovani, and thus not safe for VL treatment.

\section{CONCLUSION}

In conclusion, the in vivo activity data exhibited by AGnp provides evidence that intraperitoneal administrations to mice very efficiently reduced parasite burden in the visceral organs of acute infected BALB/c mice. A facile approach to preparing biodegradable, biocompatible, and FDA approved PLGA NPs with high drug load and encapsulation efficiency of AG using the homogenization solvent evaporation method is described. Fluorescence microscopic observation provides compelling evidence for complete internalization of FITC-PLGA NPs in macrophages, which are the host of Leishmania amastigotes. Interestingly, AG-loaded NPs turned out to be the most effective and selective in killing intracellular $L$. donovani amastigotes in macrophage cells model than first and second line antileishmanial drugs. In vitro results translate well into the in vivo model of visceral leishmaniasis, whereas phagocytic macrophage cells are the major constituent of visceral organs. Indeed, AG-loaded PLGA NPs were very efficient in reducing parasite burden in the visceral organs of acute infected BALB/c mice. Acute 
and subacute toxicity profile of AGnp were much less than the free $\mathrm{AG}$, and first and second antileishmanial drugs. In summary, we showed that AGnp has great advantages as a delivery system for AG providing controlled, effective and safe delivery for treatment of visceral leishmaniasis.

\section{ACKNOWLEDGMENT}

This study was supported by funding from Indian Council of Medical Research (ICMR) (Grant No. AMR/48/2011-ECD-I), New Delhi, India. Mr. Pallab Ghosh and Dr. Subhasish Mondal were awarded Senior Research Fellowship and Research Associateship respectively from ICMR to carry out this research work. We would like to thank Dr. Shyamol Roy of Indian Institute of Chemical Biology, Kolkata, India for his aid to procure Leishmania AG83 strain.

\section{CONFLICT OF INTERESTS}

We declare that we have no conflict of interest

\section{REFERENCES}

1. Alvar J, Vélez ID, Bern C, Herrero M, Desjeux P, Cano J, et al. Leishmaniasis worldwide and global estimates of its incidence. Plos One 2012;7:e35671.

2. Dube A, Singh N, Sundar S, Singh N. Refractoriness to the treatment of sodium stibogluconate in Indian kala-azar field isolates persist in in vitro and in vivo experimental models. Parasitol Res 2005;96:216-23.

3. Chappuis F, Sundar S, Haihe A, Ghalib H, Raijal S. Visceral leishmaniasis: what are the needs for diagnosis, treatment, and control? Nat Rev Microbiol 2007;5:873-82.

4. Burza S, Sinha PK, Mahajan R, Lima MA, Mitra G, Verma N, et al. Risk factors for visceral leishmaniasis relapse in immunocompetent patients following treatment with 20 $\mathrm{mg} / \mathrm{kg}$ liposomal amphotericin B (Ambisome) in Bihar, India. PLoS Neglected Trop Dis 2014;8:e2536.

5. Berman JJ. Treatment of leishmaniasis with miltefosine: 2008 status. Expert Opin Drug Metab Toxicol 2008;4:1209-16.

6. Kedzierski L, Sakthianandeswaren A, Curtis JM, Andrews PC, Junk PC, Kedzierska K. Leishmaniasis: current treatment and prospects for new drugs and vaccines. Curr Med Chem 2009;16:599-14.

7. Sundar S, Jha TK, Thakur CP, Sinha PK, Bhattacharya SK Injectable paromomycin for visceral leishmaniasis in India. $\mathrm{N}$ Engl J Med 2007;356:2571-81.

8. Croft SL, Olliaro P. Leishmaniasis chemotherapy-challenges, and opportunities. Clin Microbiol Infect 2011;17:1478-83.

9. Tyagi R, Sharma G, Jasuja ND, Menghani E. Indian medicinal plants as an effective antimicrobial agent. J Crit Rev 2016;3:69-71.
10. Roy P, Das S, Bera T, Mondol S, Mukherjee A. Andrographolide nanoparticles in leishmaniasis: characterization and in vitro evaluations. Int I Nanomed 2010;5:1113-21.

11. Mondal S, Roy P, Das S, Halder A, Mukherjee A, Bera T. In vitro susceptibilities of wild and drug resistant Leishmania donovani amastigote stages to andrographolide nanoparticle: the role of vitamin E derivative TPGS for nanoparticle efficacy. Plos One 2013;8:e81492.

12. Ritter U, Frischknecht F, van Zandbergen G. Are neutrophils important host cells for Leishmania parasites? Trends Parasitol 2009;25:505-10.

13. Bogdan C, Donhauser N, Döring R, Röllinghoff M, Diefenbach A, Rittig MG. Fibroblasts as host cells in latent leishmaniasis. J Exp Med 2000;191:2121-30.

14. Romero EL, Morilla MJ. Drug delivery systems against leishmaniasis? Still an open question. Expert Opin Drug Delivery 2008;5:805-23.

15. Couvreur P, Vauthier C. Nanotechnology: intelligent design to treat complex disease. Pharm Res 2006;23:1417-50.

16. Lala S, Gupta S, Sahu NP, Mandal D, Mondal NB, Moulik SP, et al. Critical evaluation of the therapeutic potential of bassic acid incorporated in oil-in-water micro emulsions and poly-D,Llactide nanoparticles against experimental leishmaniasis. J Drug Target 2006;14:171-9.

17. Espuelas MS, Legrand P, Loiseau PM, Bories C, Barratt G, Irache JM. In vitro antileishmanial activity of amphotericin B loaded in poly (epsilon-caprolactone) nanospheres. J Drug Target 2002; 10:593-9.

18. Nahar M, Jain NK. Preparation, characterization and evaluation of targeting potential of amphotericin B-loaded engineered PLGA nanoparticles. Pharm Res 2009;26:2588-98.

19. Nwaka $S$, Hudson A. Innovative lead discovery strategies for tropical diseases. Nat Rev Drug Discovery 2005;5:941-55.

20. Titus RG, Marchand M, Boon T, Louis JA. A limiting dilution assay for quantifying Leishmania major in tissues of infected mice. Parasite Immunol 1985; 7:545-55.

21. Burne BJ, Pecora R. Dynamic light scattering: with applications in chemistry, biology, and physics. Dover Publications: NY, USA; 2000.

22. Jain $\mathrm{N}$, Jain $\mathrm{R}$, Thakur N, Gupta BP, Jain DK, Banveer J, et al. Nanotechnology: a safe and effective drug delivery system. Asian J Pharm Clin Res 2010;3:159-65.

\section{How to cite this article}

- Pallab Ghosh, Subhasish Mondal, Tanmoy Bera. Preparation and characterization of andrographolide nanoparticles for visceral leishmaniasis chemotherapy: in vitro and in vivo evaluations. Int J Pharm Pharm Sci 2016;8(12):102-107. 